\section{Patrick Oswald}

\section{Liquid Crystal Growth}

$T^{\mathrm{n}}$ a system at equilibrium all is homo1 geneous in space and time at the macroscopic level. By contrast, spatiotemporal structures are frequently observed in out-of-equilibrium systems. Propagating fronts between two different phases belong to this class of phenomena. The front can be a flame, the interface between two fluids in motion (Saffman-Taylor problem), or a solid-liquid interface (growth and Mullins-Sekerka instability).

In all these examples, the problem is to predict the interface morphology as a function of its velocity.

In the following, we limit ourselves to growth fronts between two phases of the same material. The two phases can be a crystal and its melt, or a liquid crystal and its melt, or two liquid crystal phases. In metallurgy, metals and their alloys are intensively studied because most of their physical properties (mechanical, thermal and electrical) depend on the way they have been grown. In the laboratory, plastic and liquid crystals are preferred because they are transparent (and so easy to observe under the microscope) and they melt at temperatures easily accessible (near room temperature). Their symmetries and physical properties are also very varied and they allow the discovery of a variety of new phenomena such as the secondary instabilies of cellular fronts.

As a first example, we describe the free growth (at a constant temperature) of the columnar phase of a discotic liquid crystal. In this phase the disc-like molecules (figure 1) pile up in columns which form a hexagonal array. In practice, the liquid crystal always contains a small amount of impurities (the solute) so that the two phases can coexist within a small gap of temperature $T$ ranging from the solidus and the liquidus temperatures, $T_{S}$ and $T_{L}$ respectively. Experimentally, the liquid crystal is sandwiched between two glass plates spaced by a few micrometers. Figure 1 shows the growth in the plane of the hexagonal array of an initially circular germ which has nucleated in the bulk of the undercooled liquid (the free growth experiment). Its evolution depends on the undercooling $\Delta T=T_{L}-T$ where $T$ is the imposed growth temperature. Two regimes must be distinguished: the den- dritic regime at $T_{S}<T<T_{L}$ and the kinetic regime when $T<T_{S}$. In the former, the germ rapidly destabilizes along six high-symmetry directions. Then, the pattern develops into dendritic (figure 1a) or dense-branched shapes (figure $\mathrm{ib}$ ).

This instability is due to the destabilizing effect of the diffusion field of the solute that is released from the solid into the liquid (the latent heat is negligible). At large undercooling, ie at $T \ll T_{S}$, this instability disappears (absolute restabilization) because of the stabilizing effect of the surface tension that tends to minimize the perimeter length of the germ. The growth rate is then only limited by
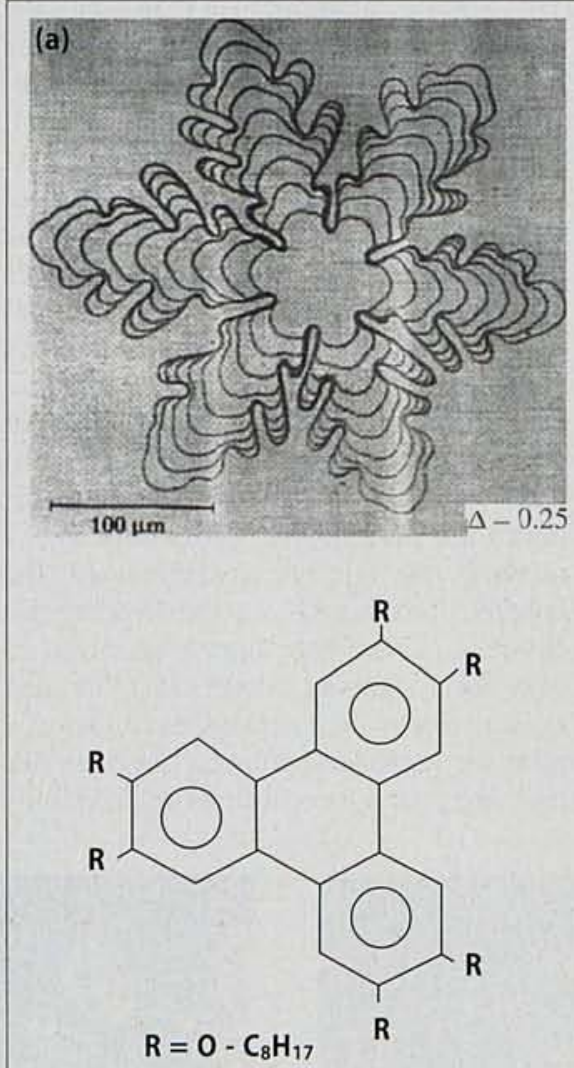

$-0.25$

the attachment kinetics of the molecules on the front: the germ then has a self-similar shape given by its kinetic anisotropy. This experiment allowed us to test recent models of dendrite selection by surface tension or kinetic anisotropy (it turns out that there exist an infinite number of dendrites that are solutions to this problem when the surface tension and the kinetic terms are set to zero) and to bring to light the morphological transitions in the diffusive and kinetic regimes untill absolute restabilization. In particular, it was possible to stress under which conditions a dendrite may be considered as two- or three-dimensional. It turns out that most dendrites in thin samples are "confined" and do not follow the expected selection rules. Their theoretical study has still to be done.

The second example concerns the nematic-isotropic interface. In a nematic phase the rod-like molecules (figure 2) tend to align parallel to each other, so that this phase possesses orientational order


Fig 1 Growth of a columnar mesophase into its melt. The liquid crystal chosen is a hexaether of triphenylene (disc-like molecule). The hexagonal array is parallel to the glass plates. The germ morphology depends on the supersaturation $\Delta$ defined as $\Delta=\left(T_{L}-T\right) /\left(T_{L}-T_{S}\right)$ where $T$ is the growth temperature, $T_{L}$ is the liquidus temperature and $T_{s}$ the solidus temperature - a At small $\Delta$ the germ shows six dendrites - b At intermediate $\Delta$ a dense-branching regime develops - c At very large $\Delta$ the germ restabilizes. Similar morphologies can be observed in the basal plane of an ice crystal growing in water SOURCE: J.C. GEMINARD 

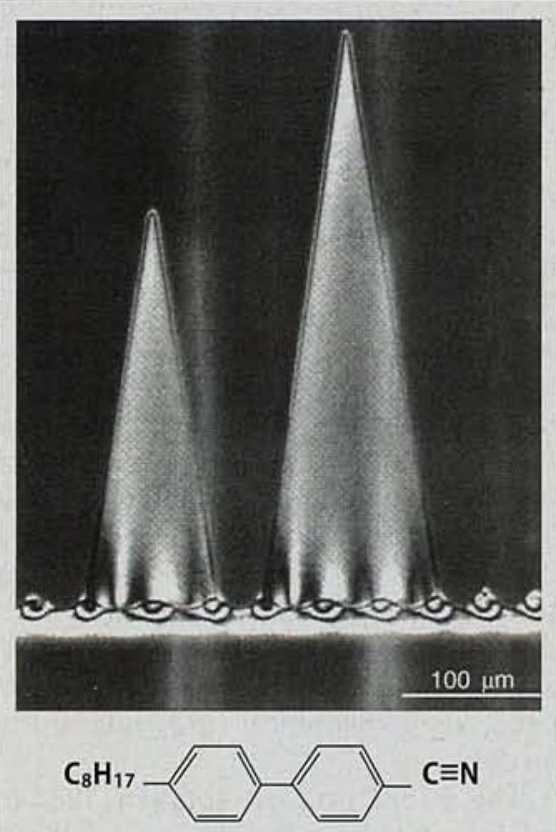

Fig 2 Mullins-Sekerka instability and cellular front at the nematic-isotropic interface observed in directional growth $\left(v=30 \mathrm{~mm} / \mathrm{s}, \mathrm{G}=20^{\circ} \mathrm{C} / \mathrm{cm}\right)$. The nematic phase is growing downwards into the isotropic liquid. The molecules have planar orientation inside a triangle, while the surrounding nematic is homeotropic (perpendicular to the plates).

A disclination line separates the two regions. The liquid crystal chosen was a cyanobiphenyl (rod-like molecule)

at long distances. The technique chosen is directional growth, consisting of moving a sample at velocity $v$ in a fixed temperature gradient $G$. After a transient, the interface freezes at velocity $-v$ in order to stay at the same temperature. The control parameters are $v$ and $G$. The experiment shows that for small velocity the interface remains flat, but for larger velocities it destabilizes into a periodic sinusoidal shape. This bifurcation is supercritical (ie the amplitude of the deformation increases slowly from zero above the onset of instability), in qualitative agreement with the linear and weakly nonlinear analysis of the Mullins-Sekerka instability, but the wavelength and the critical velocity do not follow the theorical predictions for a planar (ie flat) front. This problem is now under investigation. It was also possible to show that the front restabilizes at large velocity and above a maximal temperature gradient. In short, the full marginal stability curve is accessible with this system-this curve defines in the $(v, G)$ plane the limit between the region where the front is stable and that where the front is completely unstable. In addition, time scales for front dynamics are short, which made possible the first exploration of secondary instabilities of the cellular front (figure 2). The most studied were the "solitary modes": they consist of groups of asymmetric cells drifting through the pattern of stationary symmetric cells. These modes drift because they break the original left-right parity symmetry of the pattern. They also decrease the wavelength dispersion after their passage and allow the selection of the wavelength inside the Eckhaus band (in this band of wavelength, small phase distorsions relax diffusively, whereas outside, they grow and eventually lead to the addition or loss of a cell). Other nonlinear modes are "vacillating-breathing" modes consisting of alternating large and small cells that oscillate out of phase and a "drop instability" where small droplets of the isotropic liquid are periodically left behind in the nematic. As the velocity increases, the droplet emission shows spatio-temporal chaos. Similar secondary instabilities were then observed in other systems such as eutectics or the printer's instability of a fluid-air interface confined between two rotating cylinders. All these experiments led to a torrent of theoretical papers. Some of them used symmetry arguments to list all the possible "generic" instabilities. Others contained numerical solutions of the full equations or, more simply, projected the dynamics onto a finitedimensional dynamical system that may be studied analytically.

Finally, triangular-shaped domains (figure 2) sometimes form behind the nematic-isotropic front. This phenomenon is due to the local detachment of a virtual disclination line attached to the interface (such a line defect breaks locally the orientational order of the nematic phase).

Other interfaces that have been studied are the smectic B-smectic A and the smectic B-nematic interfaces. In smectic $A$ and $B$ phases the rod-like molecules are arranged in parallel layers and are normal to the layers. The difference between $\mathrm{A}$ and $\mathrm{B}$ phases is that in the former the layers are fluid whereas in the latter the molecules crystallize into a hexagonal array. These interfaces are faceted parallel to the smectic layers and may possess unstable orientations that are missing in the equilibrium shape of the crystal. These examples brought new insights about facet destablization and the Herring instability that develops when the front is forced to lie along a missing orientation.

Finally, we mention recent experiments in chiral materials, for instance at the cholesteric-nematic interface. A cholesteric is a spontaneously twisted nematic because of the chirality of the molecules; conversely, a nematic is an unwound cholesteric. The nematic-cholesteric transition may be observed by confining the cholesteric phase between two glass plates treated with a surface active agent that forces the molecules to anchor normally to the surface (homeotropic anchoring). Because of this anchoring, there is a frustration (it is impossible to fulfil such boundary conditions without dis-
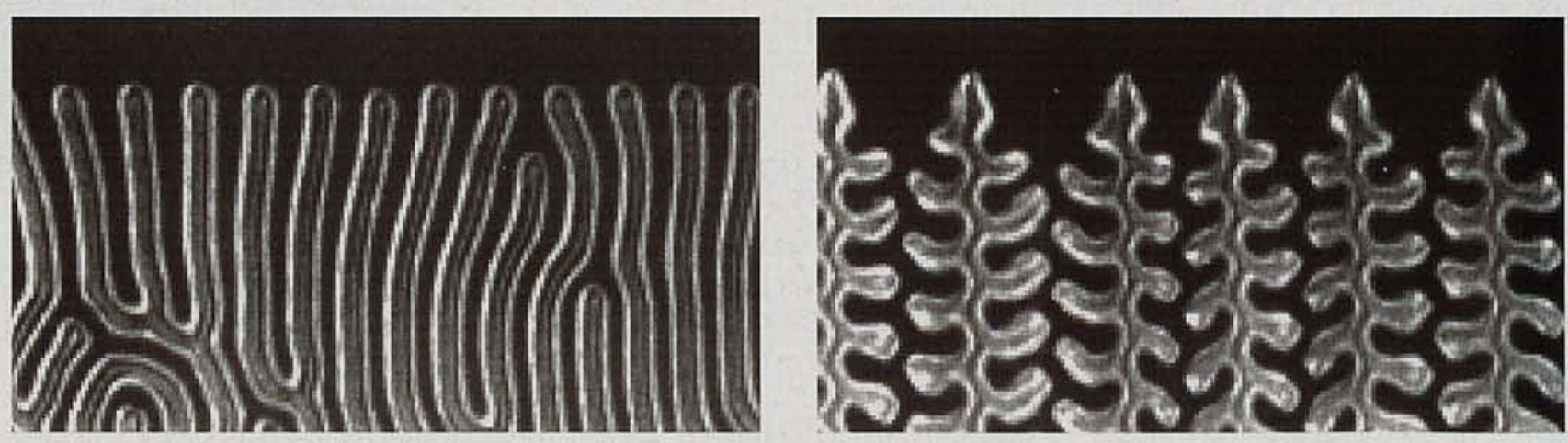

Fig 3 A cellular left to dendritic right morphological transition at a moving cholesteric-nematic interface. The cholesteric unwinds because the pitch diverges in the temperature gradient near a smectic $A$ phase 
torting the helical structure of the phase) and the nematic phase develops when the equilibrium pitch of the cholesteric phase becomes larger than the sample thickness. This transition is first order and can easily be observed when approaching a smectic phase, because the cholesteric pitch diverges at this transition. In this way, it is possible to observe the growth of the cholesteric phase into the nematic one. The main observation is that the texture of the cholesteric phase varies with the front velocity. An example of directional growth is given in figure 3 . This transition is due to a $\pi$-rotation of the cholesteric fingers (stripes) whose ends are different due to the absence of mirror symmmetry in a cholesteric. This chirality-induced morphological transition is not yet completely understood.

In conclusion, these examples show the variety of instabilities and pattern formation that arise during the growth of liquid crystals. Most phenomena observed in liquid crystals are generic and present in other systems such as metals, alloys or polymers. In particular, the questions concerning morphological transitions, confinement effects, wavelength selection, secondary instabilies and transition to chaos or turbulent states are quite general. By contrast, problems relating to chirality are more specific but could play a role in biology-does DNA have a cholesteric texture in the cell nucleus?

The author is a research director at the Ecole Normale Supérieure in Lyon in France

\section{Further Reading}

Dynamics of Curved Fronts edited by P. Pelcé

(Academic Press, New York, 1988) - "Mesophase Growth" by J. Bechhöfer in Pattern Formation in Liquid Crystals edited by A. Buka and L. Kramer (Springer, 1996) - J.C. Géminard, P. Oswald, D. Temkin, J. Malthête Europhys. Lett. 2269 (1993) - P. Oswald, J. Bechhoefer, A. Libchaber Phys. Rev. Lett. 582318 (1987) - F. Melo, P. Oswald Phys. Rev. Lett. 641381 (1990) • J. Baudry's Thèse de I'Ecole Normale Supérieure de Lyon, 1999

Colloidal dispersions - ink, paints, lubricants, cosmetics and pharmaceuticals, and foods such as milk and mayonnaiseare - are ubiquitous in everyday life and play a key role in many industrial processes. The dispersions are essentially two-phase systems, involving mesoscopic solid or liquid particles, suspended in a liquid

\author{
Jean-Pierre Hansen, England and Peter N. Pusey, Scotland
}

\title{
Phase Behaviour of Colloidal Systems
}

$T^{\text {he sizes of colloidal particles are typ- }}$ $I$ ically in the range 10 to $10^{3}$ nanometres-they are thus much larger than atoms and molecules, but small enough that Brownian motion usually dominates gravitational settling, allowing thermodynamic equilibrium to be reached.

Solid colloidal particles (to be considered here) may be mineral crystallites, like the gold solution studied by Faraday 150 years ago, or synthetic polymeric particles, like polystyrene spheres suspended in water, or amorphous polymethylmethacrylate (PMMA) particles dispersed in hydrocarbon liquids. The impenetrable mesoscopic particles usually interact via strong, attractive, shortranged van der Waals forces, which may lead to flocculation or coagulation of the colloids into gel-like structures - this led Graham to coin their name from the Greek $\kappa o \lambda \lambda \alpha$ for "glue". Flocculation may however be prevented by either steric or electrostatic stabilization. Steric stabilization is achieved by grafting polymer "brushes" on the surface of the colloidal particles, providing an elastic repulsion when two particles come so close that their "brushes" are compressed. Colloidal particles in water generally acquire a charge by dissociation of surface groups; the charged surface and microscopic co- and counter ions in solution form electric double-layers that repel strongly whenever neighbouring surfaces get closer than the Debye screening length $\lambda_{D}$.

In their studies of Brownian motion 90 years ago, Einstein and Perrin exploited already the analogy between colloids in a liquid and atoms in a gas. There are, however, significant differences between atoms and colloids, apart from the obvious change in spatial scale. In particular, the interactions between colloidal particles may be tuned, eg by the addition of salt to a dispersion of charged colloids, which leads to a reduction of $\lambda_{D}$, or by the addition of free (non-adsorbing) polymer, which leads to an effective attraction between the colloids due to the osmotic depletion effect (explained later).

These tuneable repulsive and attractive interactions between colloidal particles lead to a rich variety of phase behaviour which has been thoroughly investigated, both experimentally and theoretically. Depending on colloid concentration, and the concentration of added ions, polymers or other species, suspensions exhibit colloidal analogues of the known phases of simple molecular systems: gas, liquid, crystalline solid and glass. Colloidal crystals in suspension are easily detected by the Bragg reflection of visible light, the wavelength of which is comparable to the colloid lattice spacing, often giving them a beautiful opalescent appearance (figures 1 and 2).

\section{Binary mixtures}

Recent research has focused on colloidcolloid mixtures and colloid-polymer mixtures. The two key parameters are the size ratio $\xi=R_{B} / R_{A}$, where $R_{A}$ and $R_{B}$ denote the radii of the two species, and the degree of non-additivity of their interactions. In simple molecular systems, $\xi$ is rarely smaller than $\sim 0.5$, while interactions are almost invariably additive. In colloidal systems, by contrast, $\xi$ can take rather extreme values (as small as 0.1 or less) and colloid-polymer interactions are highly non-additive. Thus in a mixture of two species of colloid, modelled as hard spheres, the centres of two particles cannot come closer than the sum of their radii: additive interactions. Random-coil polymer molecules, however, are soft and can interpenetrate rather easily. Nevertheless, they cannot penetrate the solid colloidal particles. Thus, in a colloidpolymer mixture, the range of the colloidpolymer interaction is greater than the sum of the ranges of the self-interactions: non-additive interactions.

The importance of non-additivity of inter-species interactions is illustrated 